NIKHEF-00-020

UM-TH-00-18

\title{
New approach for calculating heavy-to-light form factors with QCD sum rules on the light-cone
}

\author{
Stefan Weinzierl ${ }^{1, a}$ and Oleg Yakovlev ${ }^{2, b}$ \\ ${ }^{1}$ NIKHEF, P.O. Box 41882, NL - 1009 DB Amsterdam, The Netherlands \\ ${ }^{2}$ Randall Laboratory of Physics, University of Michigan, Ann Arbor \\ Michigan 48109-1120, USA
}

\begin{abstract}
We suggest a new approach for calculating heavy-to-light form factors. The method is based on light cone sum rules (LCSR) and covers the whole kinematical range of momentum transfer. The derivation of the new sum rule uses a suitable combination of double and single dispersion integrals. As an example we give numerical results for the form factor $f^{+}$for the $B \rightarrow \pi$ transition.
\end{abstract}

${ }^{a}$ e-mail: stefanw@nikhef.nl

${ }^{b}$ e-mail: yakovlev@umich.edu 


\section{Introduction}

The accurate study of $\mathrm{B}$ meson decays is a main source of information for understanding $C P$ violation. We expect that the upcoming experiments will measure a variety of $\mathrm{B}$ decay properties with good precision [1]. In order to over-constrain the unitary triangle one pursues not only a measurement of the various angles, but tries as well to determine the length of the sides of the triangle. One important quantity is the CKM matrix element $\left|V_{u b}\right|$, which is proportional to the length of one side of the unitary triangle. $\left|V_{u b}\right|$ can be obtained from semileptonic B decays. There are two complementary strategies for a determination of $\left|V_{u b}\right|$, relying either on exclusive or inclusive measurements. The inclusive decay $B \rightarrow X l \nu$ can be calculated using the heavy-quark expansion [2, 3]. However one of the main obstacles here is that experimentally it is mandatory to impose restrictive cuts to suppress the background from $B \rightarrow X_{c} l \nu$ decays.

On the other hand an exclusive measurement through the channels $B \rightarrow \pi l \nu$ or $B \rightarrow$ $\rho l \nu$ is easier from an experimental point of view. It requires however a reliable theoretical calculation of the heavy-to-light transition form factors. These form factors parameterize the relevant hadronic matrix elements and are non-perturbative quantities. They can be calculated using lattice methods [4]-[8] or QCD sum rules on the light-cone. In this paper we focus on the sum rule approach.

The QCD light-cone sum rule method (LCSR) has been suggested in [9, 10, 11] and is a combination of the operator product expansion (OPE) on the light-cone [12, 13, 11] with QCD sum rule techniques [15]. For a review of the method and results we refer the reader to [16, 17.

Within the sum rule approach results for the heavy-to-light form factors corresponding to the $B \rightarrow \pi, B \rightarrow K, D \rightarrow \pi$ or $D \rightarrow K$ transitions are easily obtained from one master formula [18]- 25]. Changing the light meson from a pseudo-scalar meson to a vector meson (e.g. $\rho, \omega, K^{*}$ or $\phi$ ) the form factors of semi-leptonic or rare radiative decays of a $B$-meson into vector mesons are obtained [25, 26, 27].

To illustrate our method we focus on the form factors relevant to the $B \rightarrow \pi$ transition, defined through

$$
\left\langle\pi(q)\left|\bar{u} \gamma_{\mu} b\right| B(p+q)\right\rangle=2 f^{+}\left(p^{2}\right) q_{\mu}+\left(f^{+}\left(p^{2}\right)+f^{-}\left(p^{2}\right)\right) p_{\mu},
$$

where $p+q, q$ and $p$ denote the $B$ and $\pi$ four-momenta and the momentum transfer, respectively, and $f^{ \pm}$are the two independent form factors. If one neglects lepton masses only the form factor $f^{+}$is relevant. The standard sum rule technique starts from the correlation function of two heavy-light currents

$$
F_{\mu}(p, q)=i \int d x e^{i p \cdot x}<\pi(q)\left|T\left\{\bar{u}(x) \gamma_{\mu} b(x), m_{b} \bar{b}(0) i \gamma_{5} d(0)\right\}\right| 0>.
$$

The correlation function is then expanded near the light-cone $x^{2} \approx 0$. Each power of $x^{2}$ in the light-cone expansion leads to an additional power of $m_{b}^{2}-(p+u q)^{2}$ in the denominator. Here $u$ denotes the momentum fraction carried by one of the quarks inside the pion. The light-cone expansion is justified, provided that $(p+q)^{2}$ and $p^{2}$ are sufficiently smaller than

$m_{b}^{2}$. In particular this implies that $p^{2}<m_{b}^{2}-2 m_{b} \chi$, where $\chi$ is a scale of order $\Lambda_{Q C D}$. This limits the application of the sum rule to the kinematic region to

$$
0<p^{2}<m_{b}^{2}-2 m_{b} \chi
$$


However, in order to extract $\left|V_{u b}\right|$ one needs a prediction for the form factor $f^{+}$in the whole kinematical range $0<p^{2}<\left(m_{B}-m_{\pi}\right)^{2}$, which is much wider than (3). Several attempts have been made in the past to overcome this problem. One of the first solutions [23] consisted of extrapolating the form factor from the region $0<p^{2}<m_{b}^{2}-2 m_{b} \chi$ to the region $m_{b}^{2}-2 m_{b} \chi<p^{2}<\left(m_{B}-m_{\pi}\right)^{2}$ by assuming the functional form

$$
f^{+}\left(p^{2}\right)=\frac{f^{+}(0)}{1-a p^{2} / m_{B^{*}}^{2}+b\left(p^{2} / m_{B^{*}}^{2}\right)^{2}} .
$$

Another analysis used the fact that close to the point $p^{2}=m_{B^{*}}^{2}$ the form factor can be described by a simple pole model (see for example [28, 29, 30]), assuming vector meson dominance:

$$
f^{+}\left(p^{2}\right)=\frac{f_{B^{*}} g_{B^{*} B \pi}}{2 m_{B^{*}}\left(1-p^{2} / m_{B^{*}}^{2}\right)}
$$

The coupling $g_{B^{*} B \pi}$ can be calculated by QCD sum rules techniques, starting from the same correlation function eq. (2) and using a double dispersion relation. This additional information was used in [17] by fitting a function of the form of eq. (目) to the low $p^{2}$ behavior obtained from the standard light-cone sum rule and to a single pole model description for high $p^{2}$. A third approach was used in the analysis of [22]: The BecirevicKaidalov parameterization [31]

$$
f^{+}\left(p^{2}\right)=c_{B}\left(\frac{1}{1-p^{2} / m_{B^{*}}^{2}}-\frac{\alpha}{1-p^{2} /\left(\gamma m_{B^{*}}^{2}\right)}\right)
$$

was assumed as functional form and the parameters of this ansatz were determined from QCD sum rules, using the light-cone sum rule for $0<p^{2}<m_{b}^{2}-2 m_{b} \chi$ as well as the information on the coupling $g_{B^{*} B \pi}$. In the parameterization eq. (6) the first term corresponds to the vector meson pole, whereas the second term represents an effective contribution from all higher resonances.

All of the methods mentioned above involve ad hoc assumptions, which are difficult to justify from first principles. In addition we cannot easily quantify the error associated with these additional assumptions.

In this letter we suggest a new method for calculating heavy-to-light form factors which yields a prediction in the whole kinematical range of momentum transfer and overcomes the problem outlined above. We start from the same correlation function eq. (2). The derivation of the new sum rule is based on a combination of double and single dispersion integrals. The particular combination of double and single dispersion integrals ensures that our sum rule is valid over the whole kinematical region. As input data we need the value of $f^{+}(0)$, together with the first $l$ derivatives $f^{+(l)}\left(p^{2}\right)$ at $p^{2}=0$ (with $l$ an integer), which can be obtained (numerically) from the standard sum rule for $f^{+}\left(p^{2}\right)$. In addition we need the value of the coupling $g_{B^{*} B \pi}$, which can be obtained from the sum rule for the coupling. We derive the new sum rule to the same accuracy to which the two other sum rules are known [20, 21, 23]: to next-to-leading order in twist 2 and to leading order in twist 3 and 4 . We present numerical results for the form factor $f^{+}\left(p^{2}\right)$ for the $B \rightarrow \pi$ transition.

This paper is organized as follows: In the section 2 we introduce the new sum rule. In section 3 we give for the new sum rule the QCD corrections to twist 2 relevant for the $B \rightarrow \pi$ transition. Numerical results are given in section 4 . Section 5 contains our conclusions. 


\section{The formalism}

We write the correlation function eq. (2) in terms of invariant amplitudes $F\left(p^{2},(p+q)^{2}\right)$ and $\tilde{F}\left(p^{2},(p+q)^{2}\right)$

$$
F_{\mu}(p, q)=F\left(p^{2},(p+q)^{2}\right) q_{\mu}+\tilde{F}\left(p^{2},(p+q)^{2}\right) p_{\mu},
$$

and focus on $F\left(p^{2},(p+q)^{2}\right)$. We denote by

$$
\begin{aligned}
\sigma\left(p^{2}, s_{2}\right) & =\frac{1}{\pi} \operatorname{Im}_{s_{2}} F\left(p^{2}, s_{2}\right) \\
\rho\left(s_{1}, s_{2}\right) & =\frac{1}{\pi^{2}} \operatorname{Im}_{s_{1}} \operatorname{Im}_{s_{2}} F\left(s_{1}, s_{2}\right)
\end{aligned}
$$

the imaginary part of $F$ with respect to $s_{2}$ and the double imaginary part of $F$, respectively. Furthermore we define by

$$
\mathcal{B}_{p^{2}}=\lim _{p^{2} \rightarrow-\infty, n \rightarrow \infty,-p^{2} / n=M^{2}} \frac{1}{(n-1) !}\left(-p^{2}\right)^{n}\left(\frac{d}{d p^{2}}\right)^{n}
$$

the Borel operator with respect to the variable $p^{2}$. Hadronic representations for the spectral densities $\sigma\left(p^{2}, s_{2}\right)$ and $\rho\left(s_{1}, s_{2}\right)$ are written by singling out the ground state:

$$
\begin{aligned}
\sigma^{\text {hadr }}\left(p^{2}, s_{2}\right)= & 2 m_{B}^{2} f_{B} f^{+}\left(p^{2}\right) \delta\left(s_{2}-m_{B}^{2}\right)+\sigma^{\text {hadr }}\left(p^{2}, s_{2}\right) \Theta\left(s_{2}-s_{0}\right), \\
\rho^{\text {hadr }}\left(s_{1}, s_{2}\right)= & m_{B}^{2} m_{B^{*}} f_{B} f_{B^{*}} g_{B^{*} B \pi} \delta\left(s_{1}-m_{B^{*}}^{2}\right) \delta\left(s_{2}-m_{B}^{2}\right) \\
& +\rho^{h a d r}\left(s_{1}, s_{2}\right)\left(1-\Theta\left(s_{1}, s_{2}\right)\right) \Theta\left(s_{1}-m_{b}^{2}\right) \Theta\left(s_{2}-m_{b}^{2}\right) .
\end{aligned}
$$

Here $\Theta\left(s_{1}, s_{2}\right)$ defines the duality interval in the $\left(s_{1}, s_{2}\right)$-plane for the ground state. A convenient choice is given by the square

$$
\Theta\left(s_{1}, s_{2}\right)=\Theta\left(s_{0}-s_{1}\right) \Theta\left(s_{0}-s_{2}\right)
$$

The standard sum rule for the form factor $f^{+}\left(p^{2}\right)$ is obtained from writing a single dispersion relation for $F\left(p^{2},(p+q)^{2}\right)$ in the $(p+q)^{2}$-channel, inserting the hadronic representation and Borelizing in $(p+q)^{2}$ :

$$
\mathcal{B}_{(p+q)^{2}} F\left(p^{2},(p+q)^{2}\right)=\mathcal{B}_{(p+q)^{2}}\left(\frac{2 m_{B}^{2} f_{B} f^{+}\left(p^{2}\right)}{m_{B}^{2}-(p+q)^{2}}+\int_{s_{2}>s_{0}} d s_{2} \frac{\sigma^{h a d r}\left(p^{2}, s_{2}\right)}{s_{2}-(p+q)^{2}}\right)
$$

The Borel operator ensures that any subtraction terms which might appear will vanish after Borelization. One proceeds to replace $\sigma^{\text {had }}$ by $\sigma^{Q C D}$ and equate the r.h.s of eq. (12) to the QCD calculation of $\mathcal{B}_{(p+q)^{2}} F\left(p^{2},(p+q)^{2}\right)$. This yields the standard sum rule for $f^{+}\left(p^{2}\right)$ :

$$
f^{+}\left(p^{2}\right)=\frac{1}{2 m_{B}^{2} f_{B}} \int_{m_{b}^{2}}^{s_{0}} \sigma^{Q C D}\left(p^{2}, s_{2}\right) e^{-\frac{s_{2}-m_{B}^{2}}{M^{2}}} d s_{2}
$$


In a similar way the standard light-cone sum rule for the coupling $g_{B^{*} B \pi}$ is obtained from a double dispersion relation:

$$
\begin{aligned}
\mathcal{B}_{p^{2}} \mathcal{B}_{(p+q)^{2}} F\left(p^{2},(p+q)^{2}\right)= & \mathcal{B}_{p^{2}} \mathcal{B}_{(p+q)^{2}}\left(\frac{m_{B}^{2} m_{B^{*}} f_{B} f_{B^{*}} g_{B^{*} B \pi}}{\left(p^{2}-m_{B^{*}}^{2}\right)\left((p+q)^{2}-m_{B}^{2}\right)}\right. \\
& \left.+\int_{\Sigma} d s_{1} d s_{2} \frac{\rho^{h a d r}\left(s_{1}, s_{2}\right)}{\left(s_{1}-p^{2}\right)\left(s_{2}-(p+q)^{2}\right)}\right),
\end{aligned}
$$

where $\Sigma$ denotes the union of $s_{1}>s_{0}, s_{2}>m_{b}^{2}$ with $s_{1}>m_{b}^{2}, s_{2}>s_{0}$. Again the presence of the Borel operators ensures that any subtraction will give a vanishing contribution. The LCSR for the coupling $g_{B^{*} B \pi}$ reads:

$$
g_{B^{*} B \pi}=\frac{1}{m_{B}^{2} m_{B^{*}} f_{B} f_{B^{*}}} \int_{m_{b}^{2}}^{s_{0}} d s_{1} \int_{m_{b}^{2}}^{s_{0}} d s_{2} \rho^{Q C D}\left(s_{1}, s_{2}\right) e^{-\frac{\left(s_{1}-m_{B^{*}}^{2}\right)+\left(s_{2}-m_{B}^{2}\right)}{M^{2}}}
$$

Here we took the two Borel parameters to be equal.

To derive our new sum rule we suggest here to use a dispersion relation for $\sigma\left(p^{2}, s_{2}\right) /\left(p^{2}\right)^{l}$ in the $p^{2}$-channel (with $l$ being an integer)

$$
\sigma\left(p^{2}, s_{2}\right)=-\left.\frac{1}{(l-1) !}\left(p^{2}\right)^{l} \frac{d^{l-1}}{d s_{1}^{l-1}} \frac{\sigma\left(s_{1}, s_{2}\right)}{s_{1}-p^{2}}\right|_{s_{1}=0}+\int_{s_{1}>m_{b}^{2}} d s_{1} \frac{\left(p^{2}\right)^{l}}{s_{1}^{l}} \frac{\rho\left(s_{1}, s_{2}\right)}{s_{1}-p^{2}}
$$

and to replace $\sigma\left(p^{2}, s_{2}\right)$ in eq. (12) by the r.h.s of eq. (16). By choosing $l$ high enough the dispersion relation eq. (16) will be convergent. We obtain

$$
\begin{aligned}
& \mathcal{B}_{(p+q)^{2}} F\left(p^{2},(p+q)^{2}\right)= \\
& \mathcal{B}_{(p+q)^{2}}\left(\frac{2 m_{B}^{2} f_{B} f^{+}\left(p^{2}\right)}{m_{B}^{2}-(p+q)^{2}}+\int_{s_{1}>m_{b}^{2}, s_{2}>s_{0}} d s_{1} d s_{2} \frac{\left(p^{2}\right)^{l}}{s_{1}^{l}} \frac{\rho\left(s_{1}, s_{2}\right)}{\left(s_{1}-p^{2}\right)\left(s_{2}-(p+q)^{2}\right)}\right. \\
& \left.\quad-\left.\frac{1}{(l-1) !} \int_{s_{2}>s_{0}} \frac{d s_{2}}{s_{2}-(p+q)^{2}}\left(p^{2}\right)^{l} \frac{d^{l-1}}{d s_{1}^{l-1}} \frac{\sigma\left(s_{1}, s_{2}\right)}{s_{1}-p^{2}}\right|_{s_{1}=0}\right)
\end{aligned}
$$

Furthermore we write down a double dispersion relation for $F\left(p^{2},(p+q)^{2}\right) /\left(p^{2}\right)^{l}$ :

$$
\begin{aligned}
& \mathcal{B}_{(p+q)^{2}} F\left(p^{2},(p+q)^{2}\right)= \\
& \mathcal{B}_{(p+q)^{2}}\left(\frac{\left(p^{2}\right)^{l}}{\left(m_{B^{*}}^{2}\right)^{l}} \frac{m_{B}^{2} m_{B^{*}} f_{B} f_{B^{*}} g_{B^{*} B \pi}}{\left(p^{2}-m_{B^{*}}^{2}\right)\left((p+q)^{2}-m_{B}^{2}\right)}+\int_{\Sigma} d s_{1} d s_{2} \frac{\left(p^{2}\right)^{l}}{s_{1}^{l}} \frac{\rho\left(s_{1}, s_{2}\right)}{\left(s_{1}-p^{2}\right)\left(s_{2}-(p+q)^{2}\right)}\right. \\
& \left.\quad-\left.\frac{1}{(l-1) !} \int_{s_{2}>m_{b}^{2}} \frac{d s_{2}}{s_{2}-(p+q)^{2}}\left(p^{2}\right)^{l} \frac{d^{l-1}}{d s_{1}^{l-1}} \frac{\sigma\left(s_{1}, s_{2}\right)}{s_{1}-p^{2}}\right|_{s_{1}=0}\right)
\end{aligned}
$$

Again, by choosing $l$ high enough the dispersion integral will be convergent in the $s_{1^{-}}$ channel. The Borel operator ensures that any subtraction terms in the $s_{2}$-channel will 
vanish. Now equating the r.h.s of eq. (17) with the r.h.s of eq. (18) we obtain the sum rule

$$
\begin{aligned}
f^{+}\left(p^{2}\right)= & \frac{1}{2} \frac{\left(p^{2}\right)^{l}}{\left(m_{B^{*}}^{2}\right)^{l}} \frac{f_{B^{*}} g_{B^{*} B \pi}}{m_{B^{*}}\left(1-\frac{p^{2}}{m_{B^{*}}^{2}}\right)}-\left.\frac{1}{(l-1) !}\left(p^{2}\right)^{l} \frac{d^{l-1}}{d s_{1}^{l-1}} \frac{f^{+}\left(s_{1}\right)}{s_{1}-p^{2}}\right|_{s_{1}=0} \\
& +\frac{1}{2 m_{B}^{2} f_{B}} \int_{\Sigma^{\prime}} d s_{1} d s_{2} \frac{\left(p^{2}\right)^{l}}{s_{1}^{l}} \frac{\rho\left(s_{1}, s_{2}\right)}{s_{1}-p^{2}} e^{-\frac{s_{2}-m_{B}^{2}}{M^{2}}}
\end{aligned}
$$

where the region $\Sigma^{\prime}$ is defined by $s_{1}>s_{0}$ and $m_{b}^{2}<s_{2}<s_{0}$. This sum rule is valid in the whole kinematical range of $p^{2}$. As input data we need the first $(l-1)$ terms of the Taylor expansion of $f^{+}\left(p^{2}\right)$ around $p^{2}=0$. These parameters can be obtained numerically from the standard sum rule eq. (13). We further need the residuum at the pole $p^{2}=m_{B^{*}}^{2}$, which can be obtained from the sum rule eq. (15). The new sum rule agrees by construction with the standard sum rule eq. (13) in a Taylor expansion around $p^{2}=0$ up to the first $(l-1)$ terms. Furthermore the residuum at $p^{2}=m_{B^{*}}^{2}$ agrees with the coupling sum rule eq. (15).

We remark that the parameter $l$ plays a similar role as the Borel parameter $M^{2}$ : There is a lower limit on $l$ since the dispersion relations eq. (16) and eq. (18) have to converge. Going to higher values for $l$ will improve the convergence of the dispersion relations and suppress higher resonances in the $B^{*}$-channel. But there is also an upper limit on $l$ : The higher the value of $l$, the more derivatives of $f^{+}\left(p^{2}\right)$ at $p^{2}=0$ we have to know. At some point we start probing the region $p^{2}>m_{b}^{2}-2 \chi m_{b}$, at which the standard sum rule eq. (13) might break down. By using the sum rule with various values of $l$, say $l=1,2,3$ and by looking at the variation of the results, we can get an estimate of the uncertainty of our method.

In the case $l=0$ the second term is absent. For $l=0$ the first term corresponds to the pole model. As we will show explicitly below, the last term vanishes for the leading order twist 2, 3 and 4 contributions. The first non-vanishing contribution comes from the $\alpha_{s}$-corrections to the twist 2 contribution. This might explain the empirical fact that for some heavy-to-light transitions (like for $D \rightarrow \pi$ ) the simple pole model approximates the form factor reasonably well.

\section{The additional term}

We now consider the last term in eq. (19)

$$
f_{\text {corr }}^{+}\left(p^{2}\right)=\frac{1}{2 m_{B}^{2} f_{B}} \int_{\Sigma^{\prime}} d s_{1} d s_{2}\left(\frac{p^{2}}{s_{1}}\right)^{l} \frac{\rho\left(s_{1}, s_{2}\right)}{s_{1}-p^{2}} e^{-\frac{s_{2}-m_{B}^{2}}{M^{2}}},
$$

which has to be evaluated. The sum rules eq. (13) and eq. (15) are known in twist 2 to NLO accuracy, and in twist 3 and 4 to LO. Aiming at the same accuracy for eq. (20) we find that the LO contributions of twist two, three and four vanish. This is due to the fact that the spectral density $\rho\left(s_{1}, s_{2}\right)$ at leading order is localized along the diagonal $s_{1}=s_{2}$, whereas the integration region $\Sigma^{\prime}$ lies beyond the diagonal. At next-to-leading order the situation is different. The radiative corrections give contributions which are smeared over 
the whole $s_{1}-s_{2}$ plane, overlapping with the region $\Sigma^{\prime}$. For the twist 2 NLO contribution we find:

$$
\begin{aligned}
f_{\text {corr }}^{+}\left(p^{2}\right)=3 C_{F} \frac{\alpha_{s}}{\pi} \frac{m_{b}^{2} f_{\pi}}{2 m_{B}^{2} f_{B}}\left(\frac{p^{2}}{m_{b}^{2}}\right)^{l} \exp \left(-\frac{m_{b}^{2}-m_{B}^{2}}{M^{2}}\right)\left\{\left[\int_{1}^{\infty} d r \frac{g_{1}(r)}{(r-1)^{3}}\right.\right. \\
\left.\left.\quad-\int_{1}^{\infty} d r \frac{g_{1}^{\prime}(1)}{(r-1)^{2}}-\frac{1}{2} \int_{1}^{2} d r \frac{g_{1}^{\prime \prime}(1)}{r-1}\right]+2\left[\int_{1}^{\infty} d r \frac{g_{2}(r) \ln (r)}{(r-1)^{3}}-\int_{1}^{2} d r \frac{g_{2}^{\prime}(1)}{r-1}\right]\right\}
\end{aligned}
$$

where

$$
\begin{aligned}
g_{1}(r)= & -\int_{\frac{1+r}{r} z_{0}}^{(1+r) z_{0}} d s r e^{-\frac{b s}{1+r}}\left(\frac{1+r}{1+r+r s}\right)^{l} \frac{1}{1+r+r s-a-a r} \\
& \cdot\left(\frac{s}{1+r+s}+\ln \left(\frac{1+r+r s}{1+r+s}\right)\right), \\
g_{2}(r)= & \int_{\frac{1+r}{r} z_{0}}^{(1+r) z_{0}} d s r e^{-\frac{b s}{1+r}}\left(\frac{1+r}{1+r+r s}\right)^{l} \frac{1}{1+r+r s-a-a r} .
\end{aligned}
$$

Here we used the dimensionless variables

$$
a=\frac{p^{2}}{m_{b}^{2}}, b=\frac{m_{b}^{2}}{M^{2}}, z_{0}=\frac{s_{0}-m_{b}^{2}}{m_{b}^{2}} .
$$

Eq. (21) is written in such a form that singularities at $r \rightarrow 1$, which are present in individual contributions, cancel explicitly in the combinations inside the square brackets. The values of $g_{1}$ and $g_{2}$ and their derivatives at $r=1$ are

$$
\begin{aligned}
g_{1}(1)= & 0, \quad g_{1}^{\prime}(1)=-e^{-b z_{0}} \frac{1}{\left(1+z_{0}\right)^{l}} \frac{z_{0}^{2}}{1+z_{0}} \frac{1}{1+z_{0}-a}, \\
g_{1}^{\prime \prime}(1)= & -e^{-b z_{0}} \frac{1}{\left(1+z_{0}\right)^{l}} \frac{z_{0}^{2}}{\left(1+z_{0}\right)^{2}} \frac{1}{\left(1+z_{0}-a\right)^{2}}\left(l z_{0} a-2 z_{0} a-b z_{0} a\right. \\
& \left.-b z_{0}^{2} a-a+b z_{0}+b z_{0}^{3}+2 b z_{0}^{2}+z_{0}^{2}-l z_{0}^{2}-l z_{0}+1+2 z_{0}\right) \\
g_{2}(1)= & 0, \quad g_{2}^{\prime}(1)=-e^{-b z_{0}} \frac{1}{\left(1+z_{0}\right)^{l}} \frac{z_{0}}{1+z_{0}-a} .
\end{aligned}
$$

The integrations over $r$ and $s$ can be performed numerically.

\section{Numerical results}

We perform a numerical evaluation of the new sum rule eq. (19) for the values $l=$ $0,1,2$ and 3 . We need therefore the values of $f^{+}$and its first two derivatives at $p^{2}=0$. These numbers can be obtained from the sum rule eq. (13). The derivatives are obtained numerically according to

$$
\left(f^{+}\right)^{\prime}(0)=\frac{f^{+}(\Delta s)-f^{+}(0)}{\Delta s}, \quad\left(f^{+}\right)^{\prime \prime}(0)=\frac{f^{+}(2 \Delta s)-2 f^{+}(\Delta s)+f^{+}(0)}{(\Delta s)^{2}} .
$$




\begin{tabular}{|c|c|c|}
\hline$f_{B \pi}^{+}(0)$ & $\left(f_{B \pi}^{+}\right)^{\prime}(0)$ & $\left(f_{B \pi}^{+}\right)^{\prime \prime}(0)$ \\
\hline 0.28 & $0.014 \mathrm{GeV}^{-2}$ & $0.0014 \mathrm{GeV}^{-4}$ \\
\hline
\end{tabular}

Table 1: The form factor $f_{B \pi}^{+}$and its first derivatives at $p^{2}=0 \mathrm{GeV}$.

The sum rules eq. (13) and eq. (15) depend on various input parameters, where each parameter is only known within a certain range. A complete error analysis, where each parameter was varied within an interval, was carried out in [22]. In this letter we do not repeat such a complete error analysis. We only study the dependence on $l$ and we fix here these additional parameters to certain values. As numerical input parameters we use $m_{b}=4.7 \mathrm{GeV}, s_{0}=35 \mathrm{GeV}^{2}$ and $\alpha\left(m_{Z}\right)=0.118$. We note that the value of the pole mass is compatible with the recently updated $\overline{M S}$ mass $\bar{m}(\bar{m})=4.2 \pm 0.1$ (for a review of recent results see [32]). The same values of $m_{b}$ and $s_{0}$ have been used in the two-point sum rule for estimating the decay constant $f_{B}$. We obtained $f_{B}=183 \mathrm{MeV}$. The coefficients of the leading twist pion distribution amplitude have recently been updated in [33, 34], the coefficients of the twist three and four amplitudes can be found in [11, 18, 19, 35]. Table (1) shows the results for the form factor $f^{+}$and its first two derivatives at $p^{2}=0$ calculated from the sum rule eq. (13). We further need the $B^{*} B \pi$ coupling as input data. This value is obtained from the sum rule eq. (15) as $f_{B^{*}} g_{B^{*} B \pi}=4.4 \mathrm{GeV}$ [19, 21, 36]. With these input values we evaluate the sum rule eq. (19) for $l=0,1,2$ and 3. For the Borel parameter we use $M^{2}=10 \mathrm{GeV}^{2}$. Our results are shown in fig. (11). First of all we note a remarkable stability of the numerical results with respect to changing the number of subtractions $l$. The results for $l=1,2$ and 3 are almost identical. Secondly, the result for $l=0$ (which is the pole model plus an $\alpha_{s}$-correction term) differs at low momentum, but approaches for high momentum the results with subtractions. This corresponds to the known fact, that for the $B \rightarrow \pi$ transition the pole model does not describe the form factor accurately at low momentum. This is also shown in fig. (3). We also note that the standard sum rule for $f^{+}$eq. (13) will differ significantly from our results at high momentum. Our results for $l=1,2,3$ agree well with the parameterization given in [22]:

$$
\begin{gathered}
f_{B \pi}^{+}\left(p^{2}\right)=\frac{f_{B \pi}^{+}(0)}{\left(1-p^{2} / m_{B^{*}}^{2}\right)\left(1-\alpha_{B \pi} p^{2} / m_{B^{*}}^{2}\right)}, \\
f_{B \pi}^{+}(0)=0.28 \pm 0.05, \quad \alpha_{B \pi}=0.32 \pm_{0.07}^{0.21}
\end{gathered} .
$$

To see the difference we take $l=2$ as our main result and plot in fig. (2) the deviation

$$
R=\frac{g\left(p^{2}\right)-f_{l=2}^{+}\left(p^{2}\right)}{f_{l=2}^{+}\left(p^{2}\right)}
$$

where we take for $g$ the results $f_{l=1}^{+}, f_{l=3}^{+}$and the values according to eq. (26) with $f_{B \pi}^{+}(0)=0.28$ and $\alpha_{B \pi}=0.32$. The deviations are small.

In fig. (3) we show the sum rule results for the $B \rightarrow \pi$ form factor, eq.(19) with $l=2$ (solid line) and the pole model prediction (dotted line) in comparison to lattice results. The lattice results come from FNAL [5] (full circles), UKQCD [6] (triangles), APE [0] (full square), JLQCD [8] (open circles), and ELC [5] (semi-full circle). Taking into account the uncertainty of our result, which is estimated to be roughly $15-20 \%$ [22], we observe a satisfactory agreement with most lattice results. However, the results from 
the JLQCD collaboration lie systematically above our values. In addition, this group quotes rather small uncertainties. In general, further improvements in the accuracy of lattice calculations are welcome.

\section{Conclusions}

In this letter we have shown a method how to obtain the form factor for heavy-to-light transitions in the whole range of momentum transfer. Our method extends the QCD sum rule approach and uses a combination of single and double dispersion relations. It involves an additional (integer) parameter $l$, corresponding to the number of subtractions in one channel. We have derived the corresponding sum rule for the $B \rightarrow \pi$ transition to twist four accuracy and including radiative corrections to the twist- 2 contribution. As input data we need the first $(l-1)$ terms of the Taylor expansion of $f^{+}\left(p^{2}\right)$ around $p^{2}=0$ as well as the residuum at the pole $p^{2}=m_{B^{*}}^{2}$, which can reliable be obtained from standard sum rules. The new sum rule involves an additional term, which vanishes in leading order for the twist two, three and four contributions. We have calculated the non-vanishing next-to-leading order twist two contribution to this term. We have shown that variation of the parameter $l$ introduces only small numerical changes in the final result for the form factor, which are negligible against other uncertainties.

\section{Acknowledgements}

We are grateful to M.B. Voloshin, A.I. Vainshtein, R. Rückl for useful discussions. O.Y. acknowledges support from the US Department of Energy (DOE), S.W. would like to thank the SPhT Saclay for hospitality.

\section{References}

[1] The BaBar Physics Book, ed. P. F. Harrison and H. R. Quinn, SLAC-R-504, 1998; BELLE, Technical Design Report, KEK Report 95-1, April 1995.

[2] J. Chay, H. Georgi and B. Grinstein, Phys. Lett. B247, (1990), 399;

I. I. Bigi, N. G. Uraltsev and A. I. Vainshtein, Phys. Lett. B293, (1992), 430;

I. I. Bigi, M. Shifman, N. G. Uraltsev and A. Vainshtein, Phys. Rev. Lett. 71, (1993), 496.

[3] R. D. Dikeman and N. G. Uraltsev, Nucl. Phys. B509, (1998), 378;

I. Bigi, R. D. Dikeman and N. Uraltsev, Eur. Phys. J. C4, (1998), 453;

C. W. Bauer, Z. Ligeti and M. Luke, Phys. Lett. B479, (2000), 395;

M. Neubert, hep-ph/0006068.

[4] J. M. Flynn and C. T. Sachrajda, in Heavy Flavours II, ed. A. J. Buras and M. Lindner (World Scientific, Singapore, 1998), p. 402, hep-lat/9710057.

[5] J. M. Flynn, in Proc. of 28th ICHEP, Warsaw, ed. Z. Ajduk and A. K. Wroblewski (World Scientific, Singapore, 1996), p. 335, hep-lat/9611016. 
[6] K. C. Bowler et al. [UKQCD Collaboration], hep-lat/9910011.

[7] A. Abada et al. [APE Collaboration], Nucl. Phys. Proc. Suppl. 83-84, (2000), 268.

[8] S. Hashimoto et al. [JLQCD Collaboration], Phys. Rev. D58, (1998), 014502.

[9] I.I. Balitsky, V.M. Braun and A.V. Kolesnichenko, Nucl. Phys. B312, (1989), 509.

[10] V.L. Chernyak and A.R. Zhitnitsky, Nucl. Phys. B345, (1990), 137.

[11] V.M. Braun and I.E. Filyanov, Z. Phys. C44, (1989), 157;

V.M. Braun and I.E. Filyanov, Z. Phys. C48, (1990), 239.

[12] G.P. Lepage and S.J. Brodsky, Phys. Lett. B87, (1979), 359; Phys. Rev. D22, (1980), 2157.

[13] A.V. Efremov and A.V. Radyushkin, Phys. Lett. B94, (1980), 245; Teor. Mat. Fiz. 42, (1980), 147.

[14] V.L. Chernyak and A.R. Zhitnitsky, JETP Lett. 25, (1977), 510; Sov. J. Nucl. Phys. 31, (1980), 544; Phys. Rep. 112, (1984), 173.

[15] M.A. Shifman, A.I. Vainshtein and V.I. Zakharov, Nucl. Phys. B147, (1979), 385, 448.

[16] V. Braun, in "Heavy Flavors 8", hep-ph/9911206;

V. Braun in "Progress in Heavy Quark Physics", hep-ph/9801222;

S. Weinzierl and O. Yakovlev, J. Phys. G26, (2000), 737.

[17] A. Khodjamirian and R. Rückl, in Heavy Flavours II, ed. A.J. Buras and M. Lindner (World Scientific, Singapore, 1998), hep-ph/9801443;

R. Rückl, in 'Enrico Fermi' School of Physics, Heavy Flavor Physics, hep-ph/9810338;

S. Weinzierl and O. Yakovlev, in "Progress in Heavy Quark Physics", hepph/9712399.

[18] V.M. Belyaev, A. Khodjamirian and R. Rückl, Z. Phys. C60, (1993), 349.

[19] V.M. Belyaev, V.M. Braun, A. Khodjamirian and R. Rückl, Phys. Rev. D51, (1995), 6177.

[20] A. Khodjamirian, R. Rückl, S. Weinzierl and O. Yakovlev, Phys. Lett. B410, (1997), 275.

[21] A. Khodjamirian, R. Rückl, S. Weinzierl and O. Yakovlev, Phys. Lett. B457, (1999), 245.

[22] A. Khodjamirian, R. Rückl, S. Weinzierl, C. W. Winhart and O. Yakovlev, hep$\mathrm{ph} / 0001297$.

[23] E. Bagan, P. Ball and V.M. Braun, Phys. Lett. B417, (1998), 154.

[24] P. Ball, JHEP 9809, (1998), 005. 
[25] P. Ball, V.M. Braun and H.G. Dosch, Phys. Rev. D44, (1991), 3567.

[26] A. Ali, V.M. Braun and H. Simma, Z. Phys. C63, (1994), 437.

[27] P. Ball and V.M. Braun, Phys. Rev. D55, (1997), 5561;

P. Ball and V.M. Braun, Phys. Rev. D58, (1998), 094016.

[28] M.B. Voloshin, Sov. J. Nucl. Phys. 50, (1989), 105.

[29] G. Burdman, Z. Ligeti, M. Neubert and Y. Nir, Phys. Rev. D49, (1994), 2331.

[30] B. Grinstein and P.F. Mende, Nucl. Phys. B425, (1994), 451.

[31] D. Becirevic and A.B. Kaidalov, hep-ph/9904490.

[32] M. Beneke, in "Heavy Flavors 8", hep-ph/9911490.

[33] A. Schmedding and O. Yakovlev, hep-ph/9905392.

[34] V. M. Braun, A. Khodjamirian and M. Maul, Phys. Rev. D61, (2000), 073004.

[35] P. Ball, JHEP 9901, (1999), 010.

[36] A. G. Grozin and O. I. Yakovlev, Eur. Phys. J. C2, (1998), 721. 


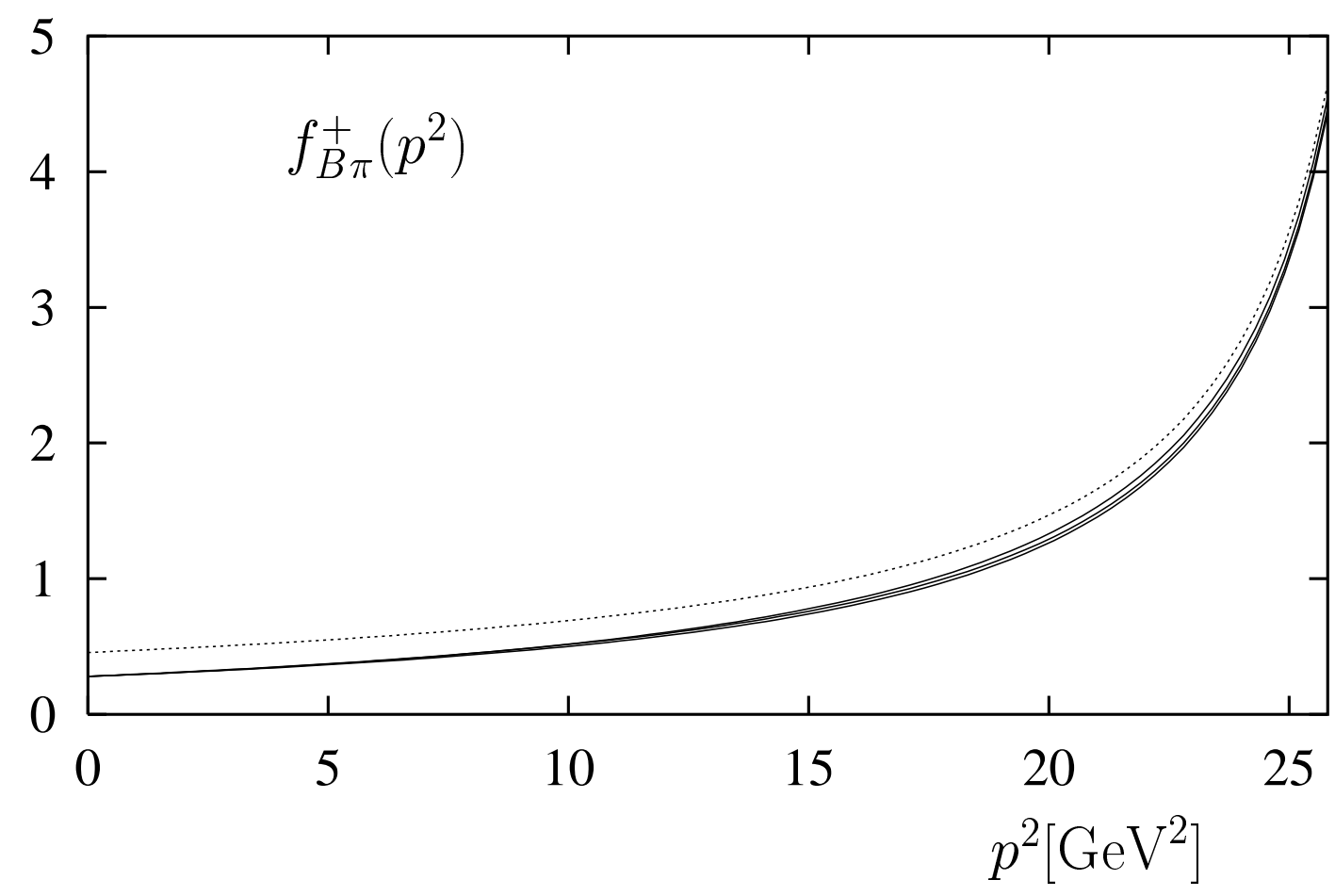

Figure 1: The results of the new sum rule for the form factor $f_{B \rightarrow \pi}^{+}\left(p^{2}\right)$ for different numbers $l$. The dotted line corresponds to $l=0$, the solid lines correspond to $l=1,2,3$.

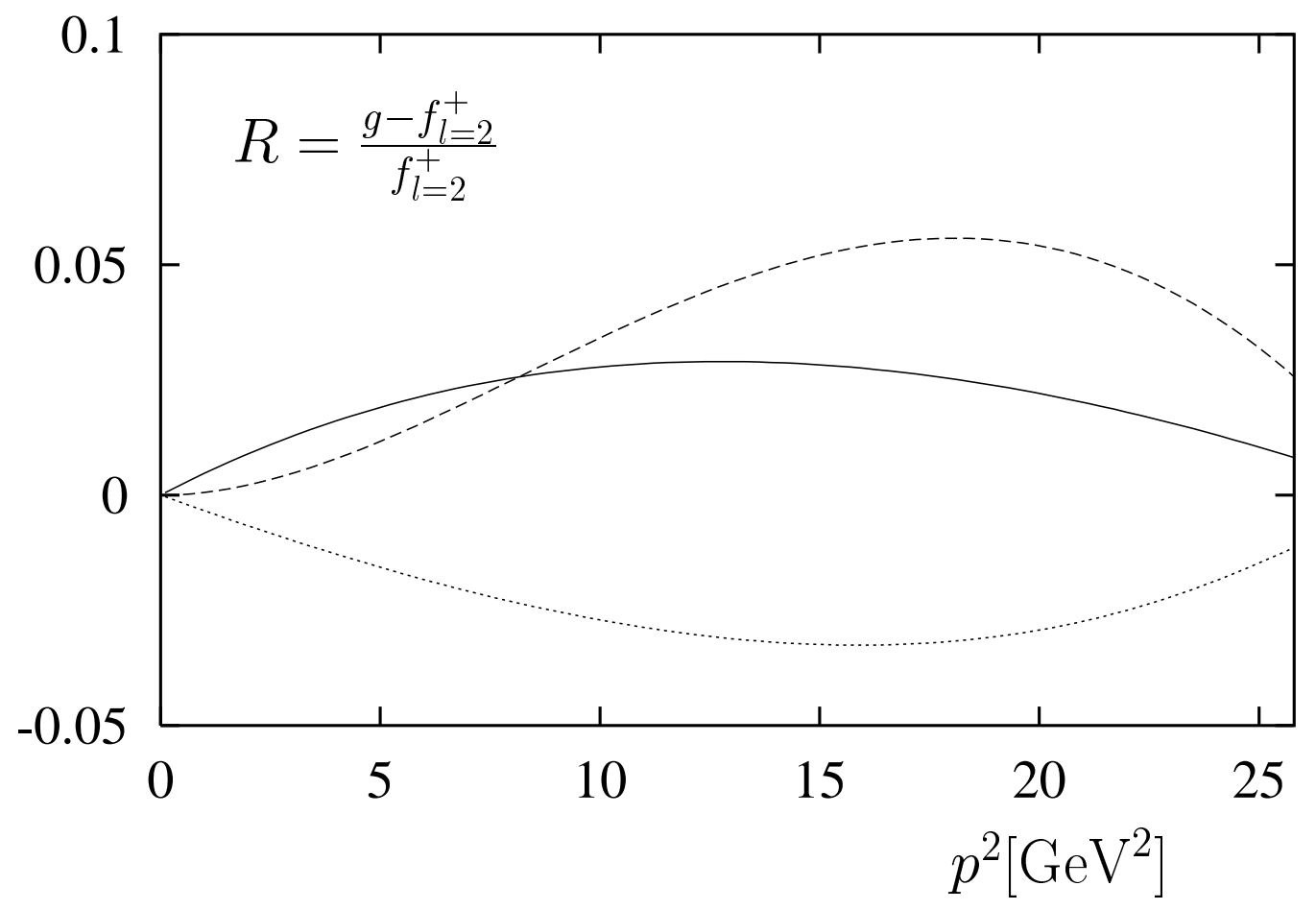

Figure 2: The relative deviation of the the numerical results with $l=1$ from $l=2$ (solid line), of $l=3$ from $l=2$ (dashed line) and of the result quoted in 22] from $l=2$ (dotted line). 


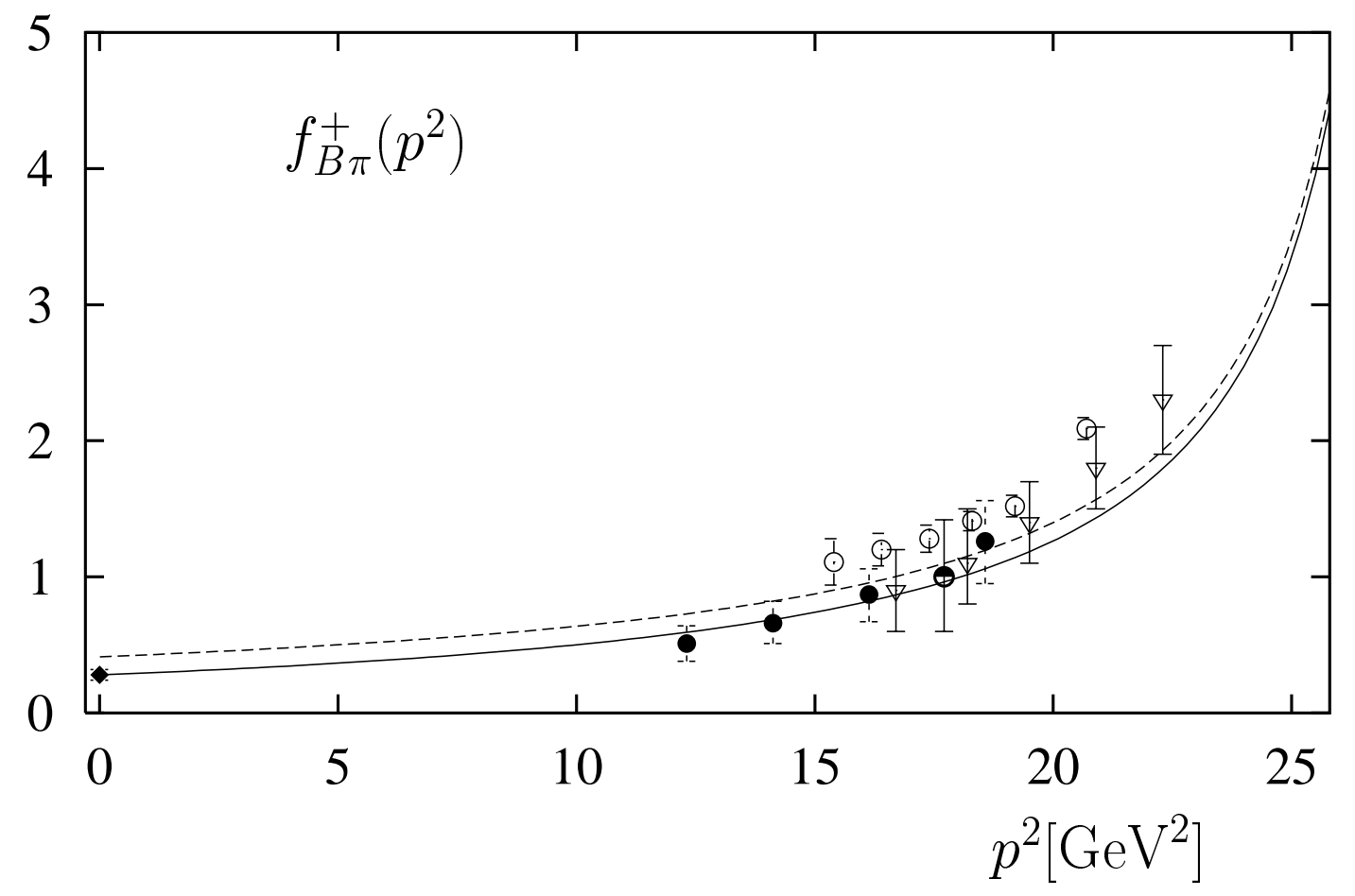

Figure 3: The sum rule results for the $B \rightarrow \pi$ form factor, eq. (19)) with $l=2$ (solid line) and the pole model result (dotted line) in comparison to lattice results. The lattice results come from FNAL [5] (full circles), UKQCD [6] (triangles), APE [7] (full square), JLQCD [8] (open circles), and ELC [5] (semi-full circle). 